\title{
Estudo comparativo do controle postural entre crianças praticantes e nõo praticantes de judô
}

\section{Comparative study of postural control among practicing and non-judo children}

\author{
Paula Zimmermann"; Simone Laral; Lilian Pinto Teixeira'; Susane Graup ${ }^{2}$ \\ 1 Departamento de Saúde, Curso de Fisioterapia, Universidade Federal do Pampa- UNIPAMPA. Uruguaiana. RS - Brasil. \\ 2 Departamento de Saúde, Curso de Educação Física, Universidade Federal do Pampa- UNIPAMPA. Uruguaiana. RS - Brasil. \\ Endereço para correspondência: \\ Simone Lara \\ Universidade Federal do Pampa- UNIPAMPA \\ Campus Uruguaiana/RS, BR 472, KM 592-CX Postal 118 \\ 97508-000 - Uruguaiana, RS [Brasil] \\ simonelara@unipampa.edu.br
}

Resumo

Objetivo: Analisar o equilíbrio postural entre crianças praticantes e não praticantes de judô. Métodos: Estudo descritivo, transversal, quantitativo, no qual foi incluída uma amostra por conveniência, formada por meninos de 06 a 09 anos de idade, sendo divididos em dois grupos: o grupo praticante de judô ( $\mathrm{N}=12)$, e o grupo não praticante $(\mathrm{N}=12)$. O equilíbrio postural das crianças foi avaliado através da Posturografia Dinâmica Computadorizada (PDC), com os testes de organização sensorial (TOS), divididos em seis condições, que avaliam a integração dos sistemas neurais visual, vestibular e somatossensorial. Resultados: Os judocas apresentaram a condição I do TOS superior ao dos não praticantes $(p=0,04)$, condição esta que avalia os três sistemas neurais responsáveis pela manutenção do equilíbrio. Conclusão: Meninos praticantes de judô apresentaram melhor interação entre os sistemas visual, vestibular e proprioceptivo e, consequentemente, melhor equilíbrio postural, quando comparados aos não praticantes.

Descritores: Equilíbrio postural; Artes marciais; Criança.

\begin{abstract}
Objective: To analyze the postural balance between practicing and non-judo children. Methods: This was a descriptive, cross-sectional, quantitative study in which a convenience sample of boys from 06 to 09 years of age was included, divided into two groups: the judo group $(\mathrm{N}=12)$ and the group practitioner $(\mathrm{N}$ $=12$ ). The postural balance of the children was evaluated through Computerized Dynamic Posturography (PDC), with sensory organization tests (TOS), divided into six conditions, which evaluate the integration of visual, vestibular and somatosensory neural systems. Results: Judokas presented condition I of TOS higher than that of non-practitioners $(\mathrm{p}=0.04)$, a condition that evaluates the three neural systems responsible for maintaining balance. Conclusions: Boys practicing judo had better interaction between the visual, vestibular and proprioceptive systems and, consequently, better postural balance, when compared to non - practitioners.
\end{abstract}

Keywords: Postural Balance; Martial Arts; Child. 


\section{Introdução}

O equilíbrio ou controle postural corresponde à habilidade de manter o centro de massa do corpo em estabilidade através da inter-relação das forças da gravidade, músculos e das forças inerciais ${ }^{1}$, sendo que, na execução de atividades do dia a dia, realização de atividade física e prática de esportes a sustentação do equilíbrio e da orientação corporal são fundamentais ${ }^{2}$. Logo, sua manutenção é obtida por meio do processamento central das aferências sensoriais compostas por informações visuais, vestibulares e proprioceptivas ${ }^{3}$.

Ao longo da infância, o processo de desenvolvimento dos sistemas sensoriais ocorre sequencialmente, no qual o amadurecimento da função proprioceptiva ocorre em torno dos 3-4 anos de idade, enquanto que os sistemas visual e vestibular parecem atingir o nível adulto aos 15-16 anos ${ }^{4}$. Além dos aspectos de maturação, sabe-se que a habilidade da criança em coordenar os sistemas de controle postural aumenta com a experiência ${ }^{5}$, logo, quanto maior forem às experiências vivenciadas pelas crianças, maior será a sua capacidade em coordenar os sistemas neurais responsáveis pelo equilíbrio. Dentre essas experiências, destaca-se a prática de modalidades esportivas na infância, como por exemplo, o judô.

O judô, como tantos outros esportes, dispõe de uma associação variada de capacidades físicas, além de pontos técnicos, táticos e psicológicos ${ }^{6}$. Suas técnicas seguem o princípio de máxima eficiência com o mínimo de esforço, baseiam-se em princípios biomecânicos que envolvem a aplicação de torque, estabilidade e equilíbrio nos momentos de inércia, centro de massa e utilização das alavancas ${ }^{7}$. Dessa forma, o tronco desempenha papel primordial durante ações de lançamento para frente em que o judoca deve descentralizar e mover o adversário para frente e para cima ${ }^{8}$. Dentre os gestos realizados no desporto, o agarrar é uma das técnicas cruciais como meio de coordenar a atenção, postura e equilíbrio do oponente e, nesse sentido, manter o equilíbrio em posição permanente é essencial para que as ações sejam efetivas durante uma disputa ${ }^{9}$.

Portanto, no decorrer da prática do judô, algumas características são treinadas e desenvolvidas, tais como equilíbrio, agilidade, força estática de membros superiores e explosão de membros inferiores ${ }^{10}$. As técnicas podem ser realizadas enquanto o competidor está em posição ortostática sobre duas pernas ou em apenas uma das pernas ${ }^{9}$. Nesse contexto, o controle eficiente do equilíbrio se faz necessário, considerando que os gestos são baseados em constantes movimentos e perturbações externas inesperadas, impostos pelo adversário com o intuito de desequilibrá-lo e derrubá-lo no combate ${ }^{11}$.

Considerando as características do esporte, Sterkowicz et al. ${ }^{9}$ encontraram adaptações importantes sobre o controle postural em judocas universitários, quando comparados a indivíduos não treinados, sugerindo a melhora desta variável nos praticantes de judô. Contudo, estudos envolvendo o controle postural de crianças praticantes de judô são limitados. Portanto, o objetivo do trabalho foi analisar o equilíbrio postural entre crianças praticantes e não praticantes de judô.

\section{Material e métodos}

Trata-se de um estudo descritivo, transversal, quantitativo, no qual foi incluída uma amostra selecionada por conveniência, formada por meninos, sendo alocados em dois grupos: um grupo praticante de judô, que está em treinamento regular em uma academia de artes marciais, com aulas semanais supervisionados por um professor responsável, e o outro não praticante de nenhuma modalidade esportiva. Os critérios de inclusão do estudo foram: sexo masculino, idade de 06 à 09 anos de idade, praticar judô regularmente por no mínimo 03 meses (para o grupo judô) e não praticar nenhuma atividade física regular (para o grupo não praticante de judô). Os critérios de exclusão foram ter alguma incapacidade física e/ou cognitiva, atestado por meio de laudo médico, que impossibilite o menino de participar do estudo. 
Cabe ressaltar que todos os preceitos éticos foram respeitados, de acordo com a Declaração de Helsinque (2008), na qual foi realizada uma reunião com os responsáveis legais de cada criança para a apresentação do projeto e, em caso afirmativo, assinavam o termo de consentimento livre e esclarecido (TCLE), autorizando a participação do menor no estudo. Os participantes assinaram o termo de assentimento. O projeto foi aprovado no Comitê de Ética e Pesquisa institucional, sob o número 2.137.073.

Após a análise de todos os critérios de inclusão descritos, participaram do trabalho 12 praticantes de judô, e 12 não praticantes. Dados sobre a idade, tempo de treinamento no judô, cor de faixa, e variáveis antropométricas foram mensuradas. A massa corporal foi avaliada por meio de balança calibrada, com o sujeito em posição ortostática, descalço e utilizando o mínimo de roupa, e a estatura foi medida através de estadiômetro, no qual o sujeito deveria permanecer em pé, ereto e com os braços estendidos ao longo do tronco ${ }^{12}$.

Esses meninos foram submetidos a um protocolo de avaliação do equilíbrio postural, no Laboratório de Avaliação do curso de Fisioterapia, da Universidade Federal do Pampa, através da Posturografia Dinâmica Computadorizada (PDC) (Sistema EquiTest ${ }^{\circledR}$ - NeuroCom International, Inc), visualizado na figura 1.

A PDC representa um instrumento padrão ouro para avaliação do equilíbrio corporal, sendo utilizada na mensuração da contribuição do sistema sensorial e motor envolvidos na manutenção do equilíbrio ${ }^{13}$. A PDC envolve o uso de perturbações do equilíbrio externo, alteração das condições superficiais ou visuais para análise da habilidade do sujeito em alcançar o controle postural ${ }^{14}$. O equipamento utilizado possui uma superfície de referência, onde o sujeito permanece em pé. Na plataforma há sensores de pressão, ativados em função do deslocamento do peso do participante sobre a planta do pé, em resposta ao deslocamento do corpo. A superfície de referência é circundada por um campo visual móvel, que sofre deslocamentos anteroposteriores ${ }^{15}$.

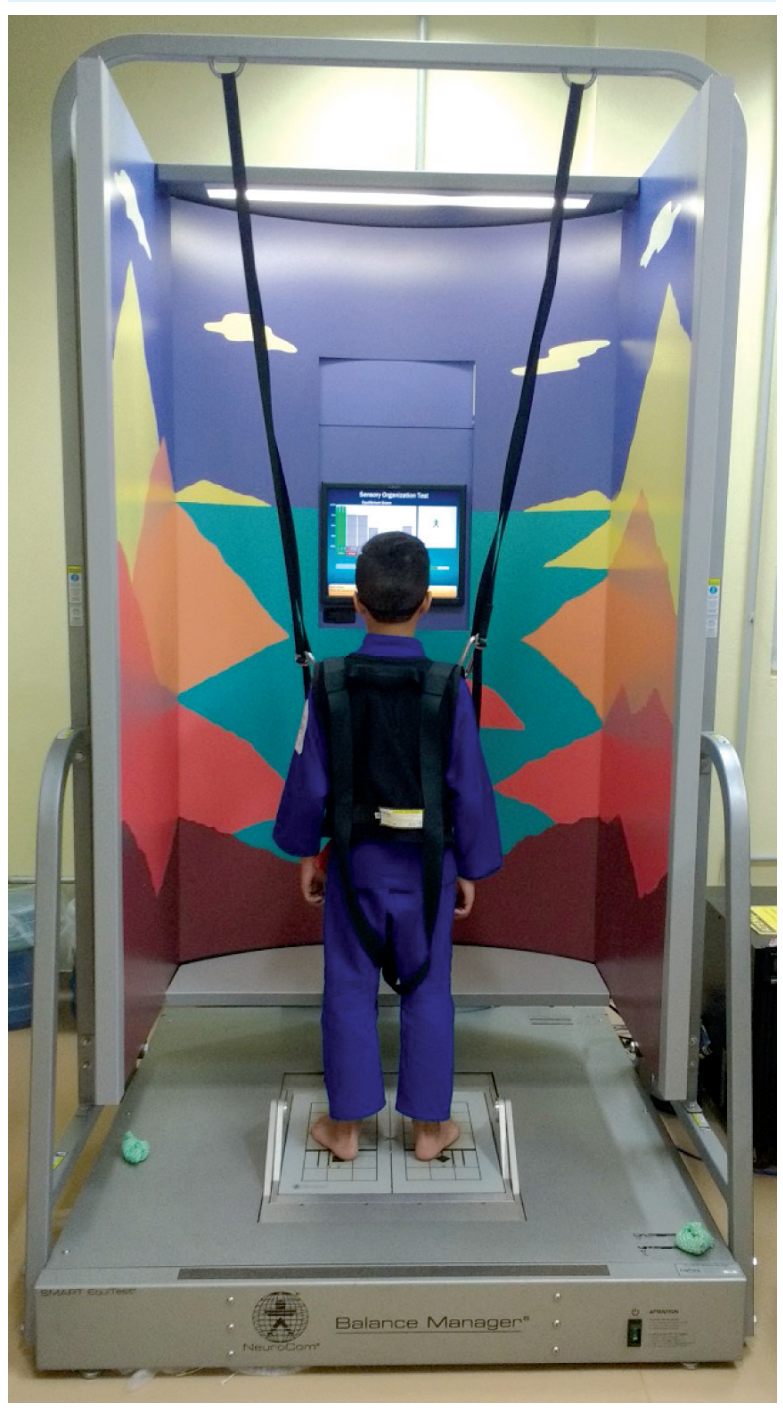

Figura l: Avaliação do equilíbrio postural por meio da Posturografia Dinâmica Computadorizada.

Fonte: os autores.

Foram realizados os testes de organização sensorial (TOS), que avaliam a coordenação e a interação dos sistemas visual, somatossensorial e vestibular durante as respostas posturais, com o intuito de quantificar a capacidade do sujeito em utilizar a integração dos diferentes sistemas para manter o equilíbrio na posição ortostáti$\mathrm{ca}^{16}$. Durante a realização dos testes, o objetivo é manter a posição vertical, o mais estável possível, durante três ensaios de 20 segundos em seis condições, mais o índice geral de equilíbrio 
(composite), indicando um melhor desempenho uma pontuação próxima de 100.

As condições oferecidas são: I, olhos abertos e plataforma fixa; II, olhos fechados e plataforma fixa; III, movimentos visuais e plataforma fixa; IV, olhos abertos e plataforma movendo-se; V, olhos fechados e plataforma movendo-se; VI, olhos abertos com ambiente e plataforma em movimento $^{17}$. Desse modo, as condições I, III e VI avaliam os sistemas visual, proprioceptivo e vestibular, enquanto que as condições II e V avaliam sistema proprioceptivo e vestibular e por fim, a condição IV avalia o sistema proprioceptivo ${ }^{18}$.

A partir das seis condições dos TOS, é possível analisar a função de cada sistema sensorial, separadamente, assim sendo ${ }^{17}$ :

- função somatosensorial: média da condição II / média da condição I;

- função visual: média da condição IV / média da condição I;

- função vestibular: média da condição V / média da condição I.

Para a análise dos dados, foi utilizado o programa Statistical Package for the Social Sciences (SPSS), versão 17.0. Todas as variáveis foram testadas quanto à sua normalidade pelo teste Shapirowilk, identificando que a amostra tinha distribuição normal. Assim, para a comparação entre os grupos aplicou-se o teste $\mathrm{t}$ para amostras independentes, e os dados foram submetidos a uma estatística descritiva (média e \pm Desvio Padrão), considerando-se estatisticamente significante $\mathrm{p}<0,05$.

\section{Resultados}

Foram incluídos 24 meninos, sendo 12 praticantes de judô e 12 não praticantes, sendo que os dados referentes à caracterização amostral estão presentes na Tabela 1, evidenciando que os grupos não possuem diferença estatística quanto à idade, massa corporal e estatura $(\mathrm{p}>0,05)$.

O tempo de treinamento do judô (no grupo dos judocas) variou entre 03 meses à 5 anos, sendo $05(20,8 \%)$ da faixa laranja, $02(8,3 \%)$ da faixa azul, $02(8,3 \%)$ da faixa branca, $02(8,3 \%)$ da faixa cinza, e $01(4,2 \%)$ da faixa amarela.

Tabela 1: Caracterização da amostra

\begin{tabular}{cccc}
\hline Variáveis & Judocas & Não judocas & $P$ \\
\hline$N$ & 12 & 12 & - \\
Idade (anos) & $7,75 \pm 1,05$ & $7,82 \pm 0,98$ & 0,69 \\
Massa $(\mathrm{Kg})$ & $36,95 \pm 9,01$ & $31,91 \pm 10,38$ & 0,21 \\
Estatura $(\mathrm{m})$ & $1,32 \pm 0,06$ & $1,29 \pm 0,06$ & 0,25 \\
\hline
\end{tabular}

Dados expressos como média e desvio padrão e desvio padrão $\pm \mathrm{DP} ; \mathrm{Kg}=$ quilos, $\mathrm{m}=$ metros.

Fonte: os autores.

Com relação à análise dos TOS (tabela 2), foi possível observar que os valores da condição I foram superiores nos judocas, quando comparados aos não judocas $(p=0,04)$, indicando um melhor equilíbrio postural nos primeiros. Nas demais condições do teste, não houve diferença significativa entre os grupos.

A tabela 3 demonstra os valores dos sistemas neurais responsáveis pela manutenção do equilíbrio postural nos praticantes e não praticantes de judô, não havendo diferenças entre os grupos.
Tabela 2: Análise descritiva dos testes de organização sensorial (TOS) de praticantes e não praticantes de judô

\begin{tabular}{|c|c|c|c|c|c|}
\hline \multirow{2}{*}{ Testes } & \multicolumn{2}{|c|}{ Judocas } & \multicolumn{2}{|c|}{ Não judocas } & \multirow[b]{2}{*}{$p$} \\
\hline & Média $\pm D P$ & IC & Média $\pm D P$ & IC & \\
\hline TOS I & $91,1 \pm 2,93$ & $89,2-92,9$ & $86,5 \pm 6,80$ & $82,2-90,8$ & $0,04^{*}$ \\
\hline TOS II & $86,8 \pm 5,99$ & $83,0-90,6$ & $86,0 \pm 4,61$ & $83,1-88,9$ & 0,73 \\
\hline TOS III & $81,2 \pm 7,06$ & $76,8-85,7$ & $81,7 \pm 7,87$ & $76,7-86,7$ & 0,87 \\
\hline TOS IV & $68,6 \pm 10,48$ & $61,9-75,3$ & $66,5 \pm 9,32$ & $60,6-72,4$ & 0,61 \\
\hline TOS V & $44,1 \pm 14,75$ & $34,7-53,5$ & $39,5 \pm 17,13$ & $28,6-50,4$ & 0,48 \\
\hline TOS VI & $37,4 \pm 13,58$ & $28,8-46,1$ & $35,2 \pm 19,34$ & $22,9-47,5$ & 0,75 \\
\hline Composite & $62,2 \pm 7,79$ & $57,3-67,2$ & $60,1 \pm 8,52$ & $54,7-65,5$ & 0,52 \\
\hline
\end{tabular}

TOS: testes de organização sensorial, expressos em percentuais (\%). DP: desvio padrão; IC: Intervalo de confiança (95\%), Teste T para amostras independentes, ${ }^{*}$ diferença estatisticamente significativa para $\mathrm{P}<0,05$ Fonte: os autores. 
Tabela 3: Anólise dos Sistemas neurais de praticantes e não praticantes de judô

\begin{tabular}{cccc}
\hline Sistemas Neurais & Judocas & Não judocas & $P$ \\
\hline Somatossensorial & $94,67 \pm 0,04$ & $98,0 \pm 0,02$ & 0,05 \\
Visual & $74,25 \pm 0,10$ & $76,58 \pm 0,09$ & 0,55 \\
Vestibular & $47,75 \pm, 015$ & $45,83 \pm 0,21$ & 0,80 \\
\hline
\end{tabular}

Valores expressos em percentuais (\%), Teste T para amostras independentes.

Fonte: os autores.

\section{Discussão}

Conforme já exposto, a PDC representa padrão ouro para avaliação da estabilidade postural ${ }^{13}$, contudo, há poucos estudos envolvendo o uso dessa ferramenta em praticantes de modalidades esportivas ${ }^{19,20}$. Em crianças saudáveis, o uso da PDC também é limitado, e nesse sentido, um estudo ${ }^{21}$ propôs valores de referência dos TOS, para crianças brasileiras, de 8 a 12 anos de idade, porém as mesmas não eram praticantes de atividade física regular. Desta forma, a presente pesquisa apresenta caráter inovador uma vez que utiliza os TOS, por meio da PDC, para analisar o controle postural de crianças brasileiras, praticantes de judô, em cotejo com as não praticantes.

Quanto à análise do controle postural, foi possível observar que os valores da condição I do TOS foram superiores nos judocas, quando comparados aos não judocas, indicando um melhor equilíbrio postural nos primeiros. Dessa forma, é válido recordar que esta condição avalia a interação entre os três sistemas sensoriais responsáveis pelo equilibrio. Sendo assim, podemos verificar que a capacidade de utilização e integração dos três sistemas sensoriais foi superior nos judocas, em comparação aos não treinados.

Os resultados do presente estudo são semelhantes ao realizado por Yoshitomi et al. ${ }^{11}$, no qual propôs analisar as respostas posturais de 30 jovens do sexo masculino, divididos em três grupos de acordo com o nível de habilidade no judô: controle (sem experiência), judocas com faixa marrom, tendo maior nível de habilidade, e judocas faixa verde, possuindo nível de habi- lidade inferior. Os autores identificaram que os atletas mais habilidosos apresentaram melhor controle do equilíbrio em relação ao grupo não praticante, revelando que a prática desse esporte favorece o desenvolvimento do controle postural. Nesse caso, sugere-se que os judocas ativam mais rapidamente a musculatura, no entanto, com maior controle nas respostas corretivas.

Os praticantes de judô cumprem exigências específicas da categoria em busca do aperfeiçoamento das técnicas e consequente melhoria da performance ${ }^{22}$. Nesse contexto, as capacidades motoras de um judoca são aprimoradas por meio de treinos voltados a aplicação de golpes, contragolpes, arremessos, desequilíbrios, esquivas e deslocamentos ${ }^{23}$, estimulando mecanorreceptores musculares, articulares e cutâneos a se adaptarem as constantes modificações na postura. Ainda, esportes de combate podem ser relevantes para a melhora do equilíbrio postural, tendo em vista que permitem ao atleta experiências em padrões posturais que incluem o controle constante do equilíbrio em função de informações sensoriais nem sempre previsíveis ou claramente disponíveis ${ }^{24}$.

Fazendo uma analogia, Brasil et al. ${ }^{24}$ identificaram em 34 participantes de jiu-jitsu adultos, que aqueles considerados experientes (tempo de prática igual ou superior a 9 anos) possuíam melhores resultados no equilíbrio dinâmico em comparação aos iniciantes (tempo de prática de pouco mais de um ano) e aos não praticantes. Portanto, o judô, assim como o jiu-jitsu, pode expor resultados similares relacionados ao equilíbrio, ao passo que ambos dispõem de movimentos em que os atletas sofrem perturbações imprevistas do oponente durante uma disputa.

Além disso, autores encontraram que o ballet e o judô podem ter impacto significativo no controle postural, considerando que ambos utilizam rotações do corpo em alta velocidade e posições em apoio unipodal, dificultando a permanência do equilíbrio. Assim, a utilização de estratégias sensório-motoras, próprias dessas modalidades, são necessárias para regulação e desenvolvimento do equilíbrio estático e dinâmico ${ }^{25,26}$. 
Nesse sentido, Perrin et al..$^{25}$ demonstraram que judocas conseguem desempenhar um melhor controle do equilíbrio em variadas circunstâncias, seja com ou sem privação sensorial ou perturbações externas, fato que evidencia a capacidade desses indivíduos em integrar os diferentes sistemas para benefício próprio, do mesmo modo, preconizam que os mesmos sejam pouco dependentes de informações visuais e mais somatossensoriais dependentes quando o objetivo é a sustentação do equilíbrio. Essa mesma afirmação foi sugerida por Itamar et al. ${ }^{27}$, no qual judocas apresentaram-se menos dependentes visualmente quando comparados a nadadores, sugerindo que os primeiros possuem um controle postural em circuito fechado mais efetivo, capaz de minimizar essa influência e esse diferencial pode ser alcançado graças ao treinamento que enfatiza as adaptabilidades sensório-motoras no atleta.

Rival et al. ${ }^{28}$ expõem que a idade de $7 \mathrm{e}$ 8 anos é marcada pela mudança na estratégia de manutenção e coordenação do equilíbrio, cujas crianças estão em processo aprendizagem das estratégias, e ainda reiteram que até os 10 anos, com os olhos fechados, as crianças não alcançaram resultados semelhantes ao grupo de adultos, sendo, portanto, mais dependentes da visão. Cabe ressaltar que essa é a faixa-etária pertencente a amostra estudada nesse estudo, e, mesmo em meio aos aspectos de maturação das estratégias posturais citados, as crianças praticantes de judô apresentaram menor dependência visual que os não praticantes, ao passo que apresentaram maior integração dos três sistemas sensoriais responsáveis pela manutenção do equilíbrio, avaliados pela condição I do TOS.

Conforme Oliveira et al..$^{29}$, são poucas as evidências que permitem afirmar se a prática de atividade física regular durante o processo de maturação e refinamento da estrutura neuro-músculo-esquelética tem maior efeito sobre o controle postural. No entanto, observamos, no presente estudo, que houve diferença no controle postural de crianças que estão em fase de maturação desses sistemas sensoriais, e percebemos o efeito benéfico da prática do judô sobre essa variável.

\section{Conclusão}

No presente estudo, ao comparar praticantes e não praticantes de judô, concluímos que os primeiros apresentaram melhor interação entre os sistemas visual, vestibular e proprioceptivo, e, consequentemente, melhor equilíbrio postural que os demais. Com base nesses achados, sugere-se que a prática de artes marciais na infância contribui de forma positiva sobre o controle postural das crianças.

Como contribuições, o estudo apresenta dados relacionados a PDC em crianças brasileiras praticantes de judô, uma vez que são escassos. Além disso, tais dados poderão contribuir para a realização de outros estudos envolvendo o controle postural na infância e sua relação com a prática de artes marciais.

\section{Referências}

1. Moraes AG, David AC, Castro OG, Marques BL, Carolino MS, Maia EM. Comparação do equilíbrio postural unipodal entre crianças e adultos. Rev Bras Educ Fís Esporte. 2014;28 (4): 571-577.

2. Duarte M, Freitas SM. Revisão sobre posturografia em uma plataforma de força para avaliação do equilíbrio. Braz. J. Phys. Ther. 2010; 14 (3): 183-92.

3. Brugnera C, Bittar RSM, Greters ME, Basta D. Efeitos da substituição vestibular vibrotactil na reabilitação vestibular - estudo preliminar. Braz. j. otorhinolaryngol. 2015; 81: 616-21.

4. Steindl R, Kunz K, Schrott-Fischer A, Scholtz AW. Effect of age and sex on maturation of sensory systems and balance control. Developmental Medicine e Child Neurology. 2006; 48(6): 477-82.

5. Graaf-Peters VB, Bakker H, Van Eykern LA, Otten B, Hadders-Algra M. Postural adjustments and reaching in 4- and 6-month-old infants: an EMG and kinematical study. Exp Brain Res. 2007;181 (4): 647-56.

6. Detanico D, Dos Santos SG. Avaliação específica no judô: uma revisão de métodos. Rev. bras. cineantropom. desempenho hum. 2012; 14 (6): 738-748. 
7. Vasconcelos DV, Cypriano D, Alves TM. Proposta de trabalho preventivo de lesões no joelho de judocas. Monografia. São Paulo: Universidade Ibirapuera; 20048.Barbado D, Lopez OA, Juan-Recio C, Montero CC, Dieen JH, Vera-Garcia FJ. Trunk Stability, Trunk Strength and Sport Performance Level in Judo. Plos One. 2016; 27;11(5):e0156267.

9. Sterkowicz S, Jaworski, J, Lech G, Pałka, T, Sterkowicz PK, Bujas P et al. Effect of Acute Effort on Isometric Strength and Body Balance: Trained vs. Untrained Paradigm. Plos One. 2016;11(5):e0155985.

10. Alonso AC, Bronzatto FE, Brech GC, Moscoli F. Estudo comparativo do equilíbrio postural entre atletas de judô e indivíduos sedentários. Rev Bras Biomec. 2008; 9 (17): 130-7.

11. Yoshitomi SK, Tanaka C, Duarte M, Lima F, Morya $\mathrm{E}$, Hazime F. Respostas posturais à perturbação externa inesperada em judocas de diferentes níveis de habilidade. Rev Bras Med Esporte. 2006;12 (3): 159-63.

12. Raphael LBM, Righi CGB. Avaliação antropométrica de crianças e adolescentes nas curvas de crescimento: uma revisão da literatura. Revista UNILUS Ensino e Pesquisa. 2016; 13 (32). 58-66.

13. Mancini M, Horak FB. The relevance of clinical balance assessment tools to differentiate balance deficits. Eur J Phys Rehabil Med. 2010; 46: 239-48.

14. Faraldo GA, Soto Varela A, Santos PS. É possível encurtar o tempo de exame em estudos de controle de postura? Acta Otorrinolaringol Esp. 2015; 66 (3): 154-8.

15. Oda DTM, Gananca CF. Posturografia dinâmica computadorizada na avaliação do equilíbrio corporal de indivíduos com disfunção vestibular. Audiol., Commun. Res. 2015; 20 (2): 89-95.

16. Amir AE, Ali AJ, Guita M, Mehdi R, Hojjat AH. The Effect of Vestibular Rehabilitation Therapy Program on Sensory Organization of Deaf Children With Bilateral Vestibular Dysfunction. Acta Med Iran. 2017; 55 (11).

17. Medeiros ÍRT, Bittar RSM, Pedalini MEB, Lorenzi MC, Kii MA, Formigoni LG. Avaliação do tratamento dos distúrbios vestibulares na criança através da posturografia dinâmica computadorizada: resultados preliminares. J Pediatr (Rio J). 2003; 79 (4): 337-342.

18. Castagno LA. A new method for sensory organization tests: the foam-laser dynamic posturographyBraz J Otorhinolaryngol. 1994; 60 (4): 287-96.
19. Oliano VJ, Teixeira LP, Lara S, Balk RS, Fagundes SG. Cumulative effect of 12 weeks of FIFA 11+ training program in addition to the handball conventional training on balance and isokinetic performance in players. Rev. bras. cineantropom. desempenho hum. 2017;19(4):406-415.

20. Barcelos B, Teixeira LP, Lara S. Análise do equilíbrio postural e força muscular isocinética de joelho em atletas de futsal feminino. Fisioter. Pesqui. 2018; 25 (1):28-34.

21. Libardoni TC, Silveira CBD, Sinhorim LMB, Oliveira AS, Santos MJD, Santos GM. Reference values and equations reference of balance for children of 8 to 12 years. Gait Posture. 2018; 60: 122-127.

22. Faquin A. Características e interelação da sensibilidade plantar e do equilíbrio de atletas e não atletas. 2005. Tese mestrado em ciências do movimento humano. Santa Catarina: Universidade de Santa Catarina; 2005.

23. Shimoda WK, Meira CM, Gomes FRF, Neiva JFO, Maia, RF. Identificação das capacidades motoras essenciais em judocas iniciantes e habilidosos. Corpoconsciência. 2007; 11: 41-53.

24. Brasil B, Chiviacowsky S, Vecchio FB, Alberton CL. Comparação do equilíbrio dinâmico entre praticantes de Brazilian Jiu-Jitsu com diferentes níveis de experiência. Rev Bras Educ Fís Esporte. 2015; 29 (4): 535-541.

25. Perrin P, Deviterne D, Hugel F, Perrot C. Judo, better than dance, develops sensorimotor adaptabilities involved inbalance control. Gait Posture. 2002; 15 (2): 187-94.

26. Costa MSS, Ferreira AS, Felicio LR. Equilíbrio estático e dinâmico em bailarinos: revisão da literatura. Fisioter. Pesqui. 2013; 20 (3): 299-305.

27. Itamar N, Schwartz D, Melzer. Controle postural: diferenças entre jovens judocas e nadadores. The Journal of Sports Medicine and Physical Fitness. 2013; 53 (5): 483-9.

28. Rival C, Ceyte H, Olivier I. Developmental changes of static standing balance in children. Neuroscience Lettrs. 2005; 11; 376 (2):133-6.

29. Oliveira TP, Santos AMC, Andrade MC, Avila AOV. Avaliação do controle postural de crianças praticantes e não praticantes de atividade física regular. Rev. Bras. Biomec. 2008; 9 (16):41-6. 